\title{
Die Mongolei auf dem Weg zu pluralistischer Demokratie und Marktwirtschaft - Die neue mongolische Verfassung von 1992
}

\author{
Von Jürgen Merz
}

\section{Einleitung}

Mit dem Zusammenbruch des kommunistischen Regimes der UdSSR bahnte sich nicht nur in Osteuropa1, sondern, der europäischen Sichtweite beinahe entrückt, auch in der Mongolischen Volksrepublik ein von verfassungsrechtlichen Änderungen begleiteter politischer Wandel an. Einerseits setzte eine Übergangsphase von einem totalitär-zentralistischen zu einem pluralistischen, rechtsstaatlich orientierten System ein. Andererseits erfuhr die ältere nationale Geschichte der Mongolei eine Rückbesinnung, die in der wiedererstarkenden Symbolkraft eines Dschinghis Khan ebenso hervortritt wie in den Erwägungen, die derzeit verwendete kyrillische durch die alte mongolische Schrift zu ersetzen. ${ }^{2}$ Ungeachtet der nationalen Renaissance sucht die Mongolei Unterstützung bei den westlichen Demokratien. Dabei kommt der Bundesrepublik deshalb eine exponierte Position zu, weil schon die DDR innerhalb des sozialistischen Staatenblocks für die Mongolei ein vorrangiger Ansprechpartner war. Daß etwa 20.000 Mongolen Deutsch sprechen und bei vielen von ihnen eine lebhafte Erinnerung an Studienaufenthalte an ostdeutschen Universitäten besteht, kann zu den wenigen erfreulichen Überresten der vom SED-Staat ausgehenden Aktivitäten gezählt werden.

Nicht nur der Bundesminister für wirtschaftliche Zusammenarbeit, der sich zuletzt im August 1992 in der Hauptstadt Ulaanbaatar aufhielt, knüpfte mit einer Finanzspritze von ca. 15 Millionen $\mathrm{DM}^{3}$ an die bisherige deutsch-mongolische Zusammenarbeit an. Auch der Deutsche Akademische Austauschdienst entsandte im September 1991 eine Delegation, um die von der DDR begonnene wissenschaftlische Kooperation unter verändertem Vorzeichen fortzusetzen. Inzwischen eröffnete der DAAD vor Ort ein Lektorat, das man mit Respekt als eine von Esprit und Tatkraft geprägte One-Man-Show bezeichnen kann. Andere Organisationen werden folgen: Die Einrichtung eines Büros der Konrad-Adenauer-Stiftung ist für das Früh jahr 1993 geplant.

1 Vgl. grundlegend dazu Peter Häberle, Verfassungsentwicklungen in Osteuropa - aus der Sicht der Rechtsphilosophie und der Verfassungslehre, AöR 117 (1992), S. 169-211.

2 Vgl. FAZ vom 7.10.1992, S. 3, und vom 31.10.1992, Beilage, ohne Seitenangabe.

3 FAZ, a.a.O. 
Bei der Vorbereitung und Durchführung einer im September 1992 wahrgenommenen Kurzzeitdozentur an der Mongolischen Staatsuniversität hatte der Verfasser Gelegenheit, sich mit Hilfe einiger weniger Materialien 4 und im Rahmen vor Ort geführter Gespräche über die neuere Rechtsentwicklung und insbesondere die im Februar 1992 in Kraft getretene Verfassung der Mongolei zu informieren.

\section{Die Mongolei bis zum Beginn des 20. Jahrhunderts}

Die Quellen des in der Abkehr vom realen Sozialismus erstarkenden mongolischen Nationalbewußtseins liegen in einer historisch gesehen relativ kurzen Periode vom frühen 13. bis zur Mitte des 14. Jahrhunderts. 5 Etwa 1206 kam es unter dem legendären Dschinghis Khan

4 Da mongolische Texte aus sprachlichen Gründen für die meisten hierzulande nur schwer zu erschließen sein werden, stehen bislang eigentlich nur eine deutsche und eine englische Übersetzung der mongolischen Verfassung - beide allerdings noch nicht veröffentlicht - sowie - ebenfalls unveröffentlichte - Unterlagen zu der internationalen Vorbereitungskonferenz "Mongolia's Transition to Democracy: The Role of the New Constitution" vom September 1991 zur Verfügung. Seit dem Sommer 1991 berichtet indessen auch die einzige englischsprachige mongolische Wochenzeitung "Mongol Messenger" über die aktuelle Entwicklung.

5 Zur mongolischen Geschichte bis in die sozialistische Ära hinein können ohne Anspruch auf Vollständigkeit folgende Titel zur weiteren Vertiefung genannt werden: Akina, Sh. (ed.), Mongolia Today, London 1991; Barthel, H., Mongolei - Land zwischen Taiga und Wüste, Gotha/Leipzig, 3. Auflage 1991; Bartow, Barry George, The policy of the Mongolian People's Republic toward China 1952 - 1973, Ann Arbor 1975; Bawden, C.R., The Modern History of Mongolia, London 1968, Neuauflage London 1989; Bira, S. / Iszamc, N., National liberation movement in Mongolia in the late 19th - early 20th centuries, Ulan Bator 1975; Bitsch, J., Zwischen China und Sibieren. Unbekannte Mongolei, Berlin 1963; Bozeman, Adda B., Politics and culture in international history, Princeton 1960; Bügd Najramdach Mongol Ard Ulsyu Tüüch (Geschichte der Mongolischen Volksrepublik), Ulaanbaatar 1956; Cheney, George Alvin, Culture change and revolution in Outer Mongolia, Ann Arbor 1977; Die Mongolische Volksrepublik. Historischer Wandel in Zentralasien (Leiter des Autorenkollektivs: Hans-Peter Vietze), Berlin (Ost) 1982; Dindub, L., A brief history of Mongolia in the autonomous period. Mongolian text with an introduction and index in English by Gombajab Hangin, Bloomington 1977; Eberhard, Wolfgang, China und seine westlichen Nachbarn. Beiträge zur mittelalterlichen und neueren Geschichte Zentralasiens, Darmstadt 1978; Ewing, Thomas Esson, Between the hammer and the anvil? Chinese and Russian policies in Outer Mongolia, Bloomington 1986; ders., Chinese and Russian policies in Outer Mongolia, 19111921, Ann Arbor 1981; Friters, G.M., Outer Mongolia and its international position, London 1951; Haggard, Mickey T., The political system of the Mongolian People's Republic, Ann Arbor 1977; Heissig, W., Die Mongolen. Ein Volk sucht seine Geschichte. Die Mongolen und die verlorenen Dokumente ihrer großen Zeit, Düsseldorf 1964; ders. (Hrsg.), Die Mongolen, 2 Bde., Innsbruck 1989; Jagchid, S. / Hyer, P., Mongolia's Culture and Society, Folkstone 1979; Korostovetz, Iwan $J$., Von Cinggis Khan zur Sowjetrepublik. Eine kurze Geschichte der Mongolei unter besonderer Berücksichtigung der neuesten Zeit, Berlin 1926; Lattimore, Owen, Nationalism and revolution in Mongolia, Leiden 1955; ders., Nomaden und Komissare. Die Mongolen gestern und heute, Stuttgart 1964; Legrand, Jacques, Le choix mongole de la féodalité au socialisme, Paris 1975; Morgan, 
nach der Einigung kleinerer Stammesgebilde zur Gründung eines einheitlichen mongolischen Staates. Der Khan und seine Nachfolger führten das nomadische Reitervolk in Eroberungszügen zu einer Machtentfaltung, die ganz Inner-, Ost- und Vorderasien und - man denke an den Vorstoß nach Ungam und Liegnitz 1241 - sogar Osteuropa umfaßte. Zu hoher Blüte gelangte das Mongolenreich mit der Verlegung seiner Hauptstadt von dem mittlerweile ruinenhaften Karalorum nach Changalyg, dem heutigen Peking, im Jahre 1259. Der Aufstand der chinesischen Bevölkerung im Jahre 1368 beendete nicht nur die von dem am Hofe Kublai Khans weilenden Marco Polo erlebte und beschriebene Herrschaft der Mongolen über China. Sie wurden vielmehr zunehmend in den Statten ihrer möchtigen Nachbarn gedrängt und letztlich Opfer einer hauptsächlich von China, aber auch vom zaristischen Rußland ausgehenden Unterdrückung. Nach und nach brachten die chinesischen Machthaber zunächst das Gebiet der heutigen inneren Mongolei und im 18. Jahrhundert schließlich das dem gegenwärtigen mongolischen Staat entsprechende Territorium unter ihre Herrschaft.

Erst 1911 riefen mongolische Fürsten im Bund mit den lamaistischen Würdenträgern des Landes im Anschluß an die bürgerliche Revolution und den Sturz der Mandschu-Dynastie

David, The Mongols, London 1986; Moses, Larry, Introduction to Mongolian history and culture, Bloomington 1985; Murphy, George G., Soviet Mongolia. A study of the oldest political satellite, Berkeley 1966; ders., The economic development of the Outer Mongolian economy in recent times, Ann Arbor 1977; Onon, Urgunge, Prichatt, Asia's first modem revolution. Mongolia proclaims its independence in 1911, Leiden 1989; Prawdin, Michael, Tschinghis Khan und sein Erbe, Stuttgart 1938; Ratchnevsky, P., Cinggis Khan. Sein Leben und sein Wirken. Wiesbaden 1983; Riasanovsky, Valentin A., Fundamental principles of Mongolian Law, Tientsin 1937 (und Bloomington 1965); Rossabi, Morris, China and Inner Asia. From 1368 to the present day, London 1975; Rupen, Robert A., Now Mongolia is really ruled. A political history of the Mongolian People's Republic 1900-1978, Stanford 1979; ders., Outer Mongolian Nationalism, 1900-1919, Ann Arbor, 1977; ders., The Mongolian People's Republic, Stanford 1966; Sagaster, Klaus, Zum Gerichtswesen in der Mongolei in der 2. Hälfte des 19. Jahrhunderts, in: Der XV. Deutsche Orientalistentag Göttingen 1961, S. 417; Salomon, Hilel Benami, China's Policy toward Outer Mongolia 1912-1920, Ann Arbor 1977; Sandag, Sh., The Mongolian People's struggle for national independence and the building of a new life. A popular scientific outline. Ulan Bator 1966; Sanders, AJ.K., The People's Republic of Mongolia. A General Reference, London 1968; ders., Mongolia. Politics, Economics, and society, London 1987; Sandorj, M., Manchu Chinese colonial rule in Northem Mongolia, London 1980; Shirendyp, B., By-passing capitalism, Ulan Bator 1968; Sirendev, B. u.a., History of the Mongolian People's Republic, Cambrigde, Mass. 1976; Slatkin, I.J., Die Mongolische Volksrepublik. Geschichtlicher Abriß, Berlin 1954; Information Mongolia. The Comprehensive Reference Source of the People's Republic of Mongolia, compiled and edited by The Academy of Sciences MPR, Oxford u.a. 1990; Taube, M. (Hrsg.), Geheime Geschichte der Mongolen. Herkunft und Aufstieg Cinggis Qans, Leipzig 1989; Thiel, Erich, Die Mongolei. Land, Volk und Wirtschaft der Mongolischen Volksrepublik, München 1958; Vietze, H.P., Der revolutionäre Weg der Mongolischen Volksrepublik zum Sozialismus. Probleme der Umgehung des kapitalistischen Entwicklungsstadiums, Berlin (Ost) 1978; Weiers, M. (Hrsg.), Die Mongolen. Beiträge zu ihrer Geschichte und Kultur, Darmstadt 1986. 
in China die Unabhängigkeit der Mongolei aus. An der Spitze des neuen, theokratisch geprägten Feudalstaates stand der Bogd Gegeen, der "lebende Buddha von Urga", als gleichermaßen weltliches und geistliches Oberhaupt. Doch schon 1915 wurde die Mongolei im Einvernehmen Rußlands und Chinas auf den Status einer unter der Oberherrschaft Chinas stehenden autonomen Provinz herabgedrückt.

Die von der Oktoberrevolution ausgelösten militärischen Auseinandersetzungen zwischen Roter Armee und Weißen Garden führten 1921 die ihren bolschwistischen Gegnern ausweichenden Truppen des baltischen Barons von Ungern-Sternberg in die Mongolei, die im Zusammenwirken mit den einheimischen Feudalherren - aber wohl auch getragen von der Bevölkerung - die chinesische Besatzungsmacht vertrieben. Der vordergründigen Befreiung folgte jedoch eine vom Baron selbst ausgeübte Pression. Zum Träger der nationalen wie sozialen Erhebung wurde schließlich die von der Sowjetunion unterstützte Mongolische Revolutionäre Volkspartei (MRVP), in deren Reihen sich auch der heute noch populäre Revolutionsführer und Volksheld Sukhbaatar6 fand. Nach der Niederlage der Weißen Garden von Ungern-Stembergs konnte schließlich am 11. Juli 1921 zum zweitenmal ein unabhängiger mongolischer Staat ausgerufen werden. Das anfangs noch de jure als - wenn auch ohnmächtige - Monarchie zu bezeichnende Staatsgebilde mit dem Bogd Gegeen als Oberhaupt wich mit dessen (natürlichen) Tod im Jahre 1924 der den faktischen Machtverhältnissen entsprechenden Mongolischen Volksrepublik unter der Führung der MRVP, ein Ergebnis, das in der Gestalt der ersten mongolischen Verfassung vom 26. November 19247 Ausdruck fand.

\section{Am Vorabend der Rechtsstaatlichkeit}

Am 30. Juni 1940 folgte eine zweite Verfassung8, die ihrerseits am 6. Juli 1960 der dritten 9 wich. Grundlegende Neuerungen im Wesen des Staates ergaben sich aufgrund dieser Änderungen indessen nicht. Erst in der zweiten Hälfte der 80er Jahre kamen dann Pläne auf, die

6 Andere Schreibweise: Suche Bator.

7 Deutsche Übersetzung in Korostovetz, Iwan J., Von Cinggis Khan zur Sowjetrepublik. Eine kurze Geschichte der Mongolei unter besonderer Berücksichtigung der neuesten Zeit, Berlin 1926, S. 344-351.

8 Deutsche Übersetzungen bei Thiel, Erich, Die Mongolei. Land, Volk und Wirtschaft der Mongolischen Volksrepublik, München 1958, S. 457-469, und Engelhardt, H., Die Verfassungen der asiatischen Länder der Volksdemokratie, Berlin (Ost) 1955.

9 Englische Übersetzungen dieser Verfassung sowie Hinweise auf weiterführende Literatur von Flanz, Gisbert H. / Karpen, Karen Shaw, Mongolian People's Republic, 1981, in: Blaustein, Albert P. / Flanz, Gisbert H. (Hrsg.), Constitutions of the Countries of the World, Binder IX, New York 1981; ferner in: Constitutions of Asian Countries, prepared by The Secretariat of the Asian-African Legal Consultative Committee New Delhi, Bombay 1968, S. 813-833; schließlich Peaslee, Amos $J$., Constitutions of Nations, Vol. II - Asia, Australia and Oceania, 3rd ed. 1966, S. 748-766. 
bisherige, 1960 entstandene und mehrmals geänderte Verfassung durch eine grundlegend neue zu ersetzen. Die damaligen Entwürfe trugen noch einen dem sozialistisıhen System verhafteten Charakter, bevor 1989 die Gegensätze zwischen neuen oppositionellen Kräften inner- wie vor allem auch außerhalb der kommunistischen 10 Einheitspartei und denjenigen, die im Glauben an einen besserungsfähigen und -würdigen realen Sozialismus verharrten, offen zutage traten. Ihren Höhepunkt erreichten die Auseinandersetzungen im März 1990, als ein Hungerstreik den Rücktritt des Zentralkomitees und neue, demokratische Strukturen der Staatsmacht erzwingen sollte.

Nachdem ein Mehrparteiensystem eingeführt worden war, konnten im Juli 1990 Wahlen stattfinden. Neben die - alte kommunistische11 - Mongolische Revolutionäre Volkspartei, die mit etwa $60 \%$ der Stimmen aus den Wahlen als Siegerin hervorging, traten neue Parteien, auf die insgesamt etwa $40 \%$ der Voten entfielen. Beispielhaft seien genannt die Mongolische Demokratische Partei (Mongolian Democratic Party - MDP), die Sozialdemokratische Partei (Social-Democratic Party - SCP), die Partei des Nationalen Fortschritts (Party of National Progress)12, ferner die Partei der nationalen Renaissance, die Liberale Partei und die Grüne Partei.

Im Anschluß an die Wahlen verfolgte die unter Führung der MRVP eingesetzte Mehrparteienregierung den Wandlungsprozeß weiter. Eine von dem im Frühjahr 1991 gewählten und derzeit amtierenden Präsidenten Otchirbat geleitete Kommission nahm ihre Tätigkeit zur Ausarbeitung einer neuen Verfassung auf. Im Rahmen der folgenden Beratungen fand vom 9. bis zum 13. September 1991 eine internationale Konferenz unter dem Titel "Mongolia's Transition to Democracy: The Role of the New Constitution" statt, bei der neben mongolischen Abgeordneten, parlamentarischen Mitarbeitern und Parteipolitikern sowie den Mitgliedern der Verfassungskommission mehrere ausländische Teilnehmer zugegen waren. Unter ihnen fanden sich Gäste aus Afghanistan, Indien, Pakistan, Vietnam, Rußland, Polen, Rumänien, Frankreich, Italien, der Bundesrepublik13 und den USA sowie ein Vertreter von Amnesty International. Auf der Grundlage der Vorarbeiten der Verfassungskommission und einer juritisch zwar unverbindlichen, faktisch aber bedeutsamen Volksbe-

$10 \mathrm{Da}$ die MRVP von mongolischer Seite mitunter zwar als revolutionäre, nicht aber kommunistische Partei angesehen wird, ist für westliche Betrachter nicht ohne weiteres nachvollziehbar, sei aber hier wenigstens ebenso angemerkt wie die Tatsache, da $B$ die MRVP of fenbar das Attribut "kommunistisch" in ihrem Namen bewußt vermieden hat.

11 Vgl. Fn. 10.

12 Information Mongolia, The Comprehensive Source of the People's Republic of Mongolia, compiled and edited by the Academy of Sciences, MPR, Oxford 1990, S. 160.

13 Prof. Dr. Theodor Schweisfurth, Heidelberg und Prof. Dr. Thomas Stein, Saarbrücken. 
fragung nahm der Große Staatschural ${ }^{14}$ im Januar 1992 einen Entwurf an15, der am 12. Februar 1992 als neue mongolische Verfassung in Kraft trat.

\section{4. Überblick über die Verfassung von 1992}

Die neue Verfassung ist unter Voranstellung einer Präambel in sechs, teilweise in verschiedene Abschnitte gegliederte Kapitel aufgeteilt, die wie folgt überschrieben sind:

Kapitel 1: Souveränität der Mongolei (Art. 1 bis 13)

Kapitel 2: Rechte und Freiheiten des menschen (Art. 14 bis 19)

Kapitel 3: Staatsaufbau der Mongolei (Art. 20 bis 56)

Abschnitt 1: Der Große Staatschural der Mongolei (Parlament)

Abschnitt 2: Staatspräsident der Mongolei

Abschnitt 3: Regierung der Mongolei

Abschnitt 4: Rechtsprechende Gewalt

Kapitel 4: Administrativ-territoriale Einheiten der Mongolei und ihre Leigung (Art. 57 bis 63)

Kapitel 5: Verfassungskontrollorgan der Mongolei - Tsets16 (Art. 64 bis 67)

Kapitel 6: Ergänzung und Änderung der Verfassung (Art. 67 bis 70)

\section{Präambel und Eingangsbestimmungen}

Während die Präambel und die Eingangsartikel der Verfassung von 1960 noch das Glaubensbekenntnis zur Oktoberrevolution und die explizite Anlehnung an die Sowjetunion beinhalten, tritt nunmehr die Hinwendung zu einer nach außen souveränen und der Vielfalt ihrer selbst bewußten Gesellschaft bürgerlicher Prägung zutage.

Wie sehr die nationale Unabhängigkeit der jungen mongolischen Demokratie ein Bedürfnis ist, wird schon in der Präambel deutlich. Im ersten Kapitel belegt dann nicht nur Art. 1 Abs. 1 den in Abkehr von der früheren Bezeichnung Mongolische Volksrepublik, oder kurz MVR, nun Mongolei genannten Staat mit den Attributen einer "unabhängige(n), souveräne(n) Republik". Darüber hinaus bezeichnet Art. 4 die territoriale Integrität und die Staatsgrenzen des Landes als unantastbar und bindet den Aufenthalt ausländischer Truppen

14 Neben der Schreibweise Chural stößt man auch auf Hural oder Khural.

15 Vgl. die Neue Zürcher Zeitung vom 16.1.1992.

16 Der in der mongolischen Fassung verwendete Begriff "Tsets" entspricht wörtlich nicht dem deutschen Begriff "Gericht". Auch den Begriff "Rat" würde man mit einem anderen Begriff ins Mongolische übersetzen, so daß hier das mongolische Wort "Tsets" unübersetzt bleiben und das damit gemeinte Organ neutral als Verfassungskontrollorgan bezeichnet werden soll. 
auf mongolischem Territorium ausdrücklich an das Erfordemis eines Gesetzes. Diese (vielleicht Ưber-) Betonung nationaler Unabhängigkeit erklärt sich überwiegend aufgrund der langen und mitunter leidvollen Geschichte des Landes in der Sandwichlage zwischen den übermächtigen Nachbarn UdSSR und China. Darüber hinaus will man die eigene Unabhängigkeit aber auch den westlichen Staaten gegenüber hervorheben, auf deren Hilfe man zwar angewiesen ist, von denen man sich andererseits verständlicherweise jedoch nicht wirtschaftlich vereinnahmen lassen möchte.

Diesen Gründen entspringt offenbar auch die im Kapitel über die Souveränität der Mongolei niedergelegte Eigentumsregelung. Werden in Art. 5 Abs. 2 noch "alle Arten des öffentlichen und privaten Eigentums" zugelassen und die Rechte des Eigentümers gesetzlich geschützt, so stößt man in Art. 6 Abs. 3 und 5 auf ein Modell, nach dem Ausländer keine Immobilien erwerben, sondern Grundstücke nur befristet zur Nutzung erhalten können.

Neben dieser außenpolitisch motivierten Einschränkung des Eigentums stößt die Privatisierung von Grund und Boden auch auf jene Grenzen, die das traditionelle, immer noch weit verbreitete und auf große Flächen frei zugänglichen Landes angewiesene Nomadentum zieht, Die Viehzüchter sind es gewohnt, mit ihren Herden von Zeit zu Zeit weiterzuziehen und stehen daher einer weitreichenden Überführung des Weidelandes in das Privateigentum einzelner wohl eher ablehnend gegenüber. Eine solche Überführung wird daher von Art. 6 Abs. 3 ausdrücklich ausgeschlossen.

\section{Staatsorganisation}

a) Parlament

\section{aa) Wahl, Zusammensetzung und Auflösung}

Höchstes Staatsorgan der Mongolei ist gemäß Art. 20 das Parlament, der Große Staatschural, dessen 76 Mitglieder in allgemeinen, freien, unmittelbaren und geheimen Wahlen auf die Dauer von vier Jahren gewählt werden. während nach dem in Kapitel 2 enthaltenen Art. 16 Nr. 9 S. 3 das aktive Wahlrecht generell für alle öffentlichen Vertretungsorgane mit der Vollendung des 18. Lebensjahrs einsetzt, liegt das Mindestalter für das passive Wahlrecht zum Parlament bei 25 Jahren. Das neugewählte Parlament wird vom Staatspräsidenten binnen 30 Tagen nach den Wahlen zu seiner konstituierenden Sitzung einberufen (Art. 27 Abs. 4 S. 1).

Das Parlament wählt aus seiner Mitte den künftig für seine Einberufung zuständigen (Art. 27 Abs. 4 S. 2) Vorsitzenden und dessen Stellvertreter für die Dauer von vier Jahren (Art. 24) und bestellt für einzelne Tätigkeiten ständige Ausschüsse (Art. 28). 
Bemerkenswert ist das in Art. 22 Abs. 2 ausgestaltete Selbstauflösungsrecht des Parlaments. Voraussetzung einer Entscheidung des Parlaments über seine Auflösung ist, daß mindestens 2/3 der Abgeordneten des Großen Staatschural sich nicht mehr in der Lage sehen, ihr Mandat fortzuführen, oder daß der Staatspräsident nach Konsultierung des Parlamentsvorsitzenden die Auflösung vorschlägt.

\section{bb) Stellung der Abgeordneten}

Gemäß Art. 23 Abs. 1 S. 1 sind die Abgeordenten Vertreter des Volkes. Nach S. 2 der Vorschrift sollen sie den Willen der gesamten Bevölkerung sowie die Staatsinteressen vertreten und achten. Wenn dieser Absatz nicht nur eine deklaratorische, sondem auch eine rechtsfolgenbewirkende Bedeutung haben soll, so muß er als Ausdruck der Hinwendung zu einer Form der repräsentativen Demokratie verstanden werden, die sowohl die Herrschaft der Parteien wie anscheinend auch einen - wohl aufgrund früherer Erfahrungen unerwünschten - übermächtigen Einfluß der Bürger in den Wahlkreisen Grenzen setzen soll.

Die Parlamentsmitglieder erhalten für ihre Abgeordnetentätigkeit, neben der sie keine anderen als die gesetzlich bestimmten Ämter und Beschäftigungen wahmehmen dürfen, eine Entschädigung aus dem Staatshaushalt (Art. 29 Abs. 1). Die Immunität soll nach Art. 29 Abs. 2 gesetzlich geschützt werden. Falls ein Abgeordneter in den Verdacht gerät, eine Straftat begangen zu haben, entscheidet das Parlament über die Aussetzung seines Mandats. Eine Verpflichtung des Churals, die Mitgliedschaft eines seiner Mitglieder aufzuheben, sieht Art. 29 Abs. 3 S. 2 darüber hinaus dann vor, wenn ein Gericht den Betroffenen - nach der Aussetzung des Mandats - einer Straftat für schuldig befunden hat. Nach dem eindeutigen Wortlaut der Bestimmung hat sich das Parlament dem Schuldspruch des Gerichts zu fügen, so daß die Aufhebung der Immunität letztlich nicht der absoluten Entscheidungsmacht des Churals unterliegt.

\section{cc) Kompetenzen und Aufgaben}

Der Aufgabenkatalog des Art. 25 weist in Abs. 1 S. 1 dem Parlament die allumfassende Berechtigung zu, alle Fragen der Innen- und Außenpolitik zu erörtem, und mündet sodann in eine Aufzählung der ausschließlich dem Chural zustehenden Kompetenzen. Dabei steht numerisch wie auch der Bedeutung entsprechend die Verabschiedung von Gesetzen an erster Stelle. Flankierend dazu steht die Uberwachung der Ausführung parlamentarischer Gesetze und sonstiger Beschlüsse des Churals. Nach Art. 25 Abs. 1 Nr. 2 und 7 bestimmt das Parlament zum einen die Grundsätze der Innen- und Außenpolitik. Zum anderen soll es die Finanz-, Kredit-, Steuer- und Geldpolitik des Staates sowie die Grundlinien der wirtschaftlichen und sozialen Entwicklung festlegen. Dies wird noch ergänzt durch die parla- 
mentarische Billigung des entsprechenden Regierungsprogramms sowie des Staatshaushalts und seiner Umsetzung.

Ferner stößt man auf eine Reihe von Befugnissen bei der Wahl anderer Staatsorgane, die aber im sachlichen Zusammenhang mit diesen behandelt werden sollen, zumal sie oft erst in den ihnen geltenden Abschnitten der Verfassung eine Konkretisierung erfahren. In eine weitere Gruppe gehören Kompetenzzuweisungen zur Gestaltung der auswärtigen Beziehungen. Hier werden etwa die Festlegung der Staatsgrenzen, die Ratifizierung und Kündigung intemationaler Verträge, die Aufnahme und der Abbruch diplomatischer Beziehungen sowie die Erklänung des Kriegszustandes ausdrücklich genannt.

Bemerkenswert ist, daß das Parlament einen Volksentscheid durchführen kann. Ein solcher vom Parlament nach dem Verfahren des Art. 27 Abs. 6 beschlossener Volksentscheid erlangt indessen nur dann Rechtsverbindlichkeit, wenn die Mehrheit der Wahlberechtigten an im teilgenommen hat. Schließlich ist es ausdrücklich Sache des Parlaments, eine Amnestie auszusprechen oder den Ausnahme- bzw. Belagerungszustand zu verhängen.

\section{b) Regierung}

\section{aa) Einsetzung, Amtsdauer, Abberufung}

Der grundsätzlich für eine Amtszeit von vier Jahren (Art. 40 Abs. 1) eingesetzten Regierung kommt nach Art. 38 Abs. 1 die Rolle des höchsten vollziehenden Organs zu. Sie besteht laut Art. 39 Abs. 1 aus dem ihr vorstehenden Ministerpräsidenten und weiteren Mitgliedem. Jedes Regierungsmitglied, also sowohl der Ministerpräsident wie auch die einzelnen, von ihm vorgeschlagenen (Art. 39 Abs. 3) Minister, wird vom Parlament (Art. 25 Abs. 1 Nr. 6, 39 Abs. 3) in sein Amt gehoben. Dabei spricht die Verfassung jeweils nur von Emennung seitens des Parlaments, scheint also nicht genau zwischen Wahl und Emennung zu unterscheiden. Jedenfalls ist keine organisatorische Aufteilung der Akte vorgesehen, etwa Wahl durch das Parlament einerseits, Emennung durch den Staatspräsidenten andererseits.

Der parlamentarischen Bestätigung unterliegt jedoch nicht nur die personelle Besetzung der Regierung, sondern auch ihre Struktur bzw. die fachliche Gliederung der Ministerien. Gemäß Art. 39 Abs. 2 unterbreitet der Ministerpräsident nach Konsultation des Staatspräsidenten seine Vorschläge zur Struktur und Zusammensetzung der Regierung jeweils dem Parlament. Die Ministerien werden dann nach Art. 46 Abs. 1 durch Gesetz gebildet.

Vor Ablauf der regulären Amtszeit von vier Jahren kann die Tätigkeit der Regierung insgesamt oder einzelner ihrer Mitglieder nach Maßgabe von Art. 43 beendet werden. Treten der 
Ministerpräsident oder mindestens die Hälfte der Minister zurück (Art. 43 Abs. 2), so muß die gesamte Regierung aus dem Amt scheiden. Der Große Staatschural hat abschließend zu entscheiden, wenn mindestens ein Viertel der Abgeordneten oder der Staatspräsident einen Antrag auf Absetzung der Regierung stellen (Art. 43 Abs. 3 und 4). Gleiches gilt für das Rücktrittsgesuch des Ministerpräsidenten. Die dem Wortlaut nach denkbare Möglichkeit, daß das Parlament das Rücktrittsgesuch ablehnt und den Ministerpräsidenten dazu zwingt, im Amt zu bleiben, ist praktisch unwahrscheinlich. Schließlich kann die Regierung gemäß Art. 44 einen Antrag stellen, ihr das Vertrauen auszusprechen, der dann vom Parlament nach dem in Art. 43 Abs. 3 festgelegten Verfahren behandelt werden muß.

\section{bb) Aufgaben und Kompetenzen}

Ähnlich wie die Kompetenzzuweisung an das Parlament leitet Art. 38 Abs. 2 die Beschreibung der Regierungsaufgaben generalklauselartig ab, wenn es heißt, daß der Regierung unter Beachtung der Gesetze die Leitung der wirtschaftlichen, sozialen und kulturellen Entwicklung des Landes obliegt. In Konkretisierung dessen folgt eine Aufzählung einzelner Aufgaben und Kompetenzen. Hierzu gehört zunächst, die landesweite Verwirklichung der "Verfassung und anderer Gesetze" sicherzustellen. Ferner stößt man auf Punkte, die man als Selbstverständlichkeiten ansehen und in der Auflistung nach deutschem Vorverständnis auch entbehren könnte, wie etwa die Durchführung der Außenpolitik, die Festigung der Verteidigungskraft und die Gewährleistung der nationalen Sicherheit, den Schutz der Menschenrechte und -freiheiten, die Sicherung der öffentlichen Ordnung sowie die Bekämpfung der Kriminalität, Maßnahmen zum Umweltschutz, zur Nutzung und zur Wiederherstellung natürlicher Ressourcen, die Erarbeitung und Ausführung von Maßnahmen zur Entwicklung der Wirtschaftszweige und der Regionen des Landes. Wohl in Abgrenzung zu den Befugnissen des Parlaments stehen die "Ausarbeitung" einer einheitlichen wissenschaftlich-technologischen Politik, der Grundlinien der wirtschaftlichen und sozialen Entwicklung, des Staatshaushalts, der Kredit- und Finanzpläne, deren Einbringung ins Parlament und die Ausführung seiner dazu ergangenen Beschlüsse. Nach dem Wortlaut der Verfassung "bestimmt" also das Parlament die entsprechenden Politikbereiche, während die Regierungstätigkeit fast idealtypisch exekutivisch beschrieben wird.

$\mathrm{Da}$ aber auch ein eigenverantwortlich wahrzunehmender, mit ausreichender Entscheidungsgewalt ausgestatteter Kernbereich an Regierungskompetenzen besteht, wird wiederum in Art. 45 vorausgesetzt. Im Rahmen ihrer Befugnisse kann die Regierung Verordnungen und Verfügungen erlassen, die in Einklang mit den Gesetzen stehen müssen. Ist letzteres nicht der Fall, so muß die Regierung selbst oder ansonsten das Parlament sie wieder außer Kraft setzen. Welche Vorgaben ein Gesetz zur Ausgestaltung und Reichweite einer Verordnung beinhalten muß, sagt die mongolische Verfassung anders als etwa Art. 80 GG indessen nicht. 


\section{c) Staatspräsident}

\section{aa) Wahl, Antritt und Beendigung des Amtes}

Staatsoberhaupt der Mongolei ist gemäß Art. 30 Abs. 1 der für vier Jahre gewählte und die Einheit des Volkes verkörpernde Präsident. Eine Wiederwahl ist nur einmal zulässig. Dem auf den ersten Blick recht komplizierten, in Art. 31 geregelten zweistufigen Wahlverfahren können sich gebürtige Bürger der Mongolei stellen, die das 45. Lebensjahr 17 vollendet und sich in den letzten fünf Jahren vor der Wahl in der Mongolei ständig aufgehalten haben. Im Parlament vertretene politische Parteien haben das Recht, allein oder gemeinsam jeweils einen Kandidaten für die Wahl zu benennen. Auf der ersten Wahlstufe findet eine von den wahlberechtigten Bürgern der Mongolei vorgenommene allgemeine, freie, unmittelbare und geheime Wahl des Präsidenten statt. Hat ein Kandidat hierbei im ersten Wahlgang die absolute Mehrheit erreicht, so soll auf der zweiten Wahlstufe das Parlament ihn als gewählten Präsidenten ansehen und per Gesetz sein Mandat anerkennen. Der neugewählte Präsident tritt gemäß Art. 32 Abs. 2 binnen 30 Tagen nach der Wahl mit der Ablegung seines Amtseides das Amt an.

Hat auf der ersten Stufe niemand im ersten Wahlgang die erforderliche Mehrheit erreicht, so müssen die beiden Kandidaten mit den besten Ergebnissen in einem zweiten Wahlgang gegeneinander antreten. Wer die absolute Mehrheit der Stimmen für sich gewinnen kann, soll sodann vom Parlament auf der zweiten Stufe wie beschrieben bestätigt werden. Kann auch im zweiten Wahlgang niemand die absolute Mehrheit erreichen, so muß das gesamte Wahlverfahren erneut begonnen werden.

Vor Ablauf der regulären Amtszeit kann der Präsident vom Parlament mit Zweidrittelmehrheit, gestützt auf ein Gutachten des Tsets, von seinem Amt enthoben werden, wenn er unter Bruch seines Eides die Verfassung verletzt bzw. seine Befugnisse mißbraucht (Art. 35 Abs. 2). Scheidet der Präsident durch Ablauf der regulären Amtszeit, Tod, Rücktritt oder Absetzung aus seinem Amt aus, so hat das Parlament binnen vier Monaten Wahlen auszuschreiben und durchzuführen. Stellvertreter des Präsidenten ist nach Maßgabe von Art. 37, der diesbezüglich eine gesetzliche Regelung verlangt, der Parlamentsvorsitzende.

17 Dies sehen die Oppositionsparteien als nachteilig an, da die meisten ihrer Mitglieder jünger sind und zunäst nur ältere, dem früheren System meistens näherstehende Kandidaten für das Präsidentenamt in Betracht kommen. 


\section{bb) Aufgaben und Kompetenzen}

Eine starke Position erfährt der Staatspräsident aufgrund des ihm in Art. 33 Abs. 1 Nr. 1 verliehenen Vetorechts. Legt er gegen einen Gesetzes- oder sonstigen Beschluß des Parlaments ganz oder teilweise ein Veto ein, so bleiben die entsprechenden Teile nur in Kraft, wenn der Einspruch des Präsidenten von mindestens 2/3 der Abgeordneten zurückgewiesen wird. Weiter ist der Staatspräsident von Bedeutung für die Einsetzung und Abberufung der Regierung, insofern er dem Parlament - grundsätzlich nach Konsultierung der Mehrheitspartei - den Kandidaten für das Amt des Ministerpräsidenten oder auch die Entlassung der Regierung vorschlägt.

Unklar erscheint die Machtverteilung nach Art. 33 Abs. 1 Nr. 3. Einerseits kann der Präsident der Regierung gegenüber Direktiven erlassen. Andererseits bedürfen nach dem Wortlaut der Vorschrift Erlasse, die in Wahmehmung dieser Befugnis ergehen, ebenso wie andere Anordnungen der Gegenzeichnung durch den Ministerpräsidenten. Ferner kommen dem Präsidenten etwa die völkerrechtliche Vertretung des Landes im internationalen Verkehr, das Begnadigungsrecht, die Erklärung der Mobilmachung und auch - bei Verhinderung des Parlaments - die vorläufige Verhängung des Ausnahme- und Belagerungszustandes zu.

\section{d) Rechtsprechende Gewalt}

Die Rechtsprechung wird nach Art. 47 Abs. 1 ausschließlich von Gerichten wahrgenommen, die mit unabhängigen, nur dem Gesetz unterworfenen Richtern besetzt sind. Über die Einhaltung dieser Anforderungen soll gemäß Art. 49 Abs. 3 der "Oberste Rat des Richterstandes" 18 wachen, der selbst kein Recht spricht, sondern ausschließlich für die Auswahl der Richter, die Wahrung ihrer Rechte und andere für die Aufrechterhaltung einer unabhängigen Rechtsprechung bedeutsame Angelegenheiten zuständig ist. Die Zusammensetzung des Rates und das von ihm zu verfolgende Verfahren legt die Verfassung nicht selbst fest, sondern überläßt es in Art. 49 Abs. 5 dem einfachen Gesetzgeber.

Das Gerichtssystem ist nach Art. 49 Abs. 1 dreistufig mit dem Obersten Gericht an der Spitze und diesem untergeordnet entsprechend den Verwaltungseinheiten Aimag-Gerichten auf der mittleren und Sum-Gerichten auf der untersten Stufe. Ausdrücklich erwähnt werden das im Status einem Aimag-Gericht vergleichbare Gericht der Hauptstadt sowie diesem untergeordnete, auf einer Stufe mit den Sum-Gerichten stehende Stadtbezirksgerichte. die Verfassung sieht bei Einhaltung dieser Struktur ferner die Einrichtung von Fachgerichten wie - wohl beispielhaft aufgeführten - Straf-, Zivil- und Verwaltungsgerichten vor.

Englische Übersetzung: "General Council of Courts". 
Art. 50 benennt die Streitigkeiten, für die das Oberste Gericht zuständig ist. Dieses soll einmal - soweit gesetzlich vorgesehen - erstinstanzlich über "Strafsachen und andere Rechtsstreitigkeiten" entscheiden, darüber hinaus die Entscheidungen unterer Gerichte als Appellations- und Kassationsgericht überprüfen. Neben diesen eher geläufigen Verfahrensarten stehen die Überprüfung und Entscheidung von Angelegenheiten, die die Menschenrechte und Grundfreiheiten betreffen und ihm vom Tsets oder dem Generalstaatsanwalt übermittelt wurden. Schließlich ists das Oberste Gericht für die Erstellung offizieller Auslegungen zur korrekten Anwendung aller Gesetze zuständig, ausdrücklich jedoch nicht für gleiches hinsichtlich der Verfassung.

\section{e) Verwaltungsaufbau}

Die größte territoriale Verwaltungseinheit der Mongolei heißt Aimag und ist ihrerseits in mehrere Sums aufgeteilt, während auf der untersten Stufe die Bags als kleinste Verwaltungsdistrikte bestehen (Art. 57 Abs. 1). Die Hauptstadt Ulaanbaatar, die in diesem Zusammenhang besonders erwähnt wird, entspricht einem Aimag, ihre Stadtbezirke sind den Sums, die einzelnen Wohnviertel (choroos) hingegen den Bags vergleichbar.

Bei der Ausgestaltung der Verwaltungstätigkeit beschreitet man einen Mittelweg, der Elemente der örtlichen Selbstverwaltung mit solchen staatlicher bzw. zentraler Leitung verbinden soll (Art. 59). So bestehen auf den Ebenen der Aimags und Sums, aber auch noch darunter als Selbstverwaltungsorgane Versammlungen gewählter Vertreter, während jeweils die staatlichen Befugnisse ein Vorsteher19 wahmimmt, der auf Vorschlag des jeweiligen Selbstverwaltungsorgans in den Aimags vom Ministerpräsidenten und auf den untergeordneten Ebenen vom Vorsteher des nächst höheren Distriktes ernannt wird (Art. 60).

Der Vorsteher führt als reines Exekutivorgan gleichermaßen die Beschlüsse des jeweiligen Selbstverwaltungsorgans wie auch die Vorgaben der übergeordneten Verwaltungsebenen bis hin zu Parlaments- und Regierungsakten aus. Demgegenüber wird das Selbstverwaltungsorgan zur Wahmehmung der auf seiner Ebene liegenden lokalen Belange und eines Kreises von Aufgaben tätig, den man etwa als Pflichtaufgaben 20 beschreiben könnte.

Im Falle eines Konfliktes zwischen einem Selbstverwaltungsorgan und dem ihm zugeordneten Vorsteher genießt letzterer auf den ersten Blick aufgrund des ihm in Art. 61 eingeräumten Vetorechts scheinbar die stärkere Position. Widerspricht er einem Beschluß der Versammlung, so kann er seinen Rücktritt beantragen, wenn die Versammlung sein Veto

19 Deutsche Übersetzung: "Hauptmann"; englische Übersetzung: "governor".

20 Die deutsche Übersetzung spricht sinngemäß von Aufgaben, die landesweit oder im Rahmen der übergeordneten Verwaltungseinheit erfüllt werden müssen. 
zurückweist und er dennoch dieAusführung des Beschlusses nicht für möglich hält. Die Konsequenzen dieser Regelung sind indessen nicht ganz klar. Fraglich ist, ob der Vorsteher sich bei Fortführung seines Amtes weigem kann, einem ihm widerstrebenden Beschluß auszuführen, oder ob ihm gegebenenfalls nur die Möglichkeit des Rücktritts bleibt. Stellt man in Rechnung, daß der Vorsteher zwar auf Vorschlag der Versammlung bestimmt wurde, aber nicht ihr, sondern der übergeordneten staatlichen Verwaltungsinstanz verantwortlich ist (Art. 61 Abs. 1 S. 2), müßte man die erste Lösung befürworten. Ein transparentes Modell zur Konfliktbewältigung gibt die Verfassung jedenfalls nicht vor.

\section{f) Verfassungskontrollorgan - Tsets 21}

Zur Wahrung ihrer selbst sieht die mongolische Verfassung ein Verfassungskontrollorgan vor, von dessen neun auf sechs jahre vom Parlament ernannten Mitgliedern jeweils drei von letzterem selbst, vom Staatspräsidenten und vom Obersten Gericht vorgeschlagen werden. Diese wählen wiederum aus ihrer Mitte bei einmalig zulässiger Wiederwahl für jeweils drei Jahre einen Vorsitzenden. Die Möglichkeit einer vorzeitigen Absetzung des Vorsitzenden oder eines sonstigen Mitglieds sieht Art. 65 Abs. 4 für den Fall vor, daß der Betroffene ein Gesetz verletzt. Die Absetzung kann dann durch das Parlament erfolgen, allerdings nur aufgrund der Entscheidung des Tsets selbst und des Organs, das die Wahl des Betroffenen vorgeschlagen hat. Demnach kann lediglich die vorzeitige Amtsenthebung einzelner Mitglieder erzwungen werden. Bei einem Konflikt zwischen dem Tsets als solchem und den anderen Staatsorganen behält er offenbar die Oberhand.

Ein Verfahren vor dem Tsets können nach Art. 66 Abs. 1 per Antrag in Gang setzen: das Parlament, der Staatspräsident, das Oberste Gericht und der Generalstaatsanwalt. Unter welchen genaueren Voraussetzungen der Tsets auf Betreiben der Bürger tätig wird, erschließt sich nicht eindeutig aus der Verfassung. Heißt es in der deutschen Übersetzung sinngemäß "aufgrund der von den Bürgern vorgebrachten Verfassungsbeschwerden", so sagt die englische "on its own initiative on the basis of petitions and informations received from the citizens" und liegt damit näher an der mongolischen Fassung. Inwieweit der Tsets einen Ermessensspielraum besitzt und in welchem Maße die Bürger einen Anspruch auf Rechtsschutz haben, bleibt offen, wird aber sicher Gegenstand eines Gesetzes über das Verfassungskontrollorgan sein.

Zu den einzelnen in Art. 66 Abs. 2 aufgeführten Streitigkeiten, für deren Behandlung er zuständig ist, gibt der Tsets teilweise Gutachten ab, auf deren Grundlage das Parlament entscheidet, teilweise steht das letzte Wort aber auch dem Verfassungsgericht selbst zu. In die zweite Gruppe fällt zunächst die Beurteilung der Verfassungmäßigkeit der von Parla-

21 Vgl. oben Fn. 16. 
ment, Staatspräsident und Regienung vorgenommenen Akte. Ferner entscheidet der Tsets darüber, ob ein Volksentscheid oder die Feststellung eines Ergebnisses bei den Parlamentsund Präsidentschaftswahlen mit der Verfassung in Einklang steht. In all diesen Fällen trifft der Tsets nach nochmaliger Überprüfung die abschließende Entscheidung, wenn das von ihm dem Parlament übermittelte Gutachten von diesem zurückgewiesen wird. Befindet der Tsets, daß der betreffende Akt verfassungswidrig ist, so hat dies dessen Nichtigkeit zur Folge.

Um Gutachten im eigentlichen Sinne handelt es sich, wenn der Tsets dem Parlament eine Stellungnahme darüber abgibt, ob die Gründe für eine Amtsenthebung des Präsidenten, des Parlamentsvorsitzenden oder des Ministerpräsidenten oder die Aufhebung eines Abgeordnetenmandats vorliegen. In diesen Fällen soll das Parlament schon nach anderen Verfassungsartikeln selbst die endgültige Entscheidung treffen.

\section{g) Gesetzgebung und Verfassungsänderung}

Die Gesetzesinitiative haben der Staatspräsident, die Regierung oder einzelne Abgeordnete des Parlaments (Art. 26 Abs. 1). Dieses entscheidet bei Anwesenheit von mindestens zwei Dritteln der Abgeordneten grundsätzlich mit der einfachen Mehrheit der Anwesenden. Abweichungen von dieser Mehrheitsregelung können nicht nur in die Verfassung aufgenommen werden, sondern schon durch einfachen Gesetzesbeschluß zustande kommen (Art. 27 Abs. 6).

Nach Art. 68 Abs. 1 i.V.m. Art. 26 Abs. 1 können wiederum der Staatspräsident, die Regierung und der einzelne Abgeordnete sowie der Tsets Vorschläge zur Verfassungsänderung einbringen. Laut Art. 69 Abs. 1 bedarf die Änderung einer Mehrheit von drei Vierteln der Parlamentsmitglieder. Eine Besonderheit stellt die in Art. 68 Abs. 2 vorgesehene Möglichkeit des Volksentscheids über einen Entwurf zur Verfassungsänderung dar, der auf Verlangen von zwei Dritteln der Abgeordneten stattfinden muß. Fraglich dürfte indessen das Verhältnis zwischen dem Volksentscheid einerseits und der parlamentarischen Entscheidung andererseits sein, das alternativ, aber auch kumulativ verstanden werden könnte. Für ersteres spricht der Verweis auf Art. 26 Abs. 1 Nr. 16, nach dem sich eine zur Abstimmung gestellte Angelegenheit dem Mehrheitsvotum entsprechend erledigt.

\section{Grundrechte}

Zu Beginn des Grundrechtskapitels löst die Beschränkung des Gleichheitsgrundsatzes auf die sich recht- bzw. gesetzmäßig in der Mongolei befindenden Personen in Art. 14 Abs. 1 Befremden aus. Inwiefern dieser Limitienung wirklich ein entsprechendes Grundrechtsver- 
ständnis zugrunde liegt, bleibt allerdings abzuwarten. Es ist durchaus vorstellbar, daß den Verfassem die Tragweite der Formulierung nicht gänzlich bewußt war.22

Konkretisierungen des Gleichheitssatzes findet man sowohl in Art. 14 Abs. 2 wie auch in Art. 16 Nr. 11. Die erste Vorschrift enthält ein aus vielen Verfassungen und internationalen Vereinbarungen bekanntes Diskriminierungsverbot, das eine Benachteiligung aufgrund von Merkmalen wie Abstammung, Rasse etc. untersagt. Die zweite Bestimmung formuliert die Gleichberechtigung von Mann und Frau im öffentlichen und familiären Bereich.

Bei der Auflistung einzelner Grundrechte im Katalog des Art. 16 fällt zunächst auf, daß nach dieser Bestimmung nur mongolische Bürger die aufgeführten Grundrechte und Freiheiten genießen, nicht aber Ausländer, deren Rechte und Pflichten einer besonderen, am Grundsatz der Gegenseitigkeit orientierten Regelung in Art. 18 vorbehalten bleiben.

Das elementare Recht auf Leben gewährt die Verfassung zwar in Art. 16 Nr. 1 S. 1. Schon aus dem nächsten $S a t z$ geht indessen hervor, daß sich die mongolischen Verfassungsschöpfers nicht zu einer Abschaffung der Todesstrafe durchringen konnten und diese von Verfassungs wegen explizit erhalten bleibt.

Das in der Bundesrepublik derzeit nicht mehrheitsfähige und ihrer traditionellen Grundrechtsdogmatik nicht entsprechende Recht auf ein Leben in einer gesunden und sicheren Umwelt sowie auf den Schutz vor Umweltverschmutzung und des ökologischen Gleichgewichts fand in die neue mongolische Verfassung (Art. 14 Nr. 2) dagegen Eingang. Der tatsächliche Wert dieses Rechts läßt sich angesichts einer wohl noch fehlenden und in ihrer Entwicklung noch nicht absehbaren Grundrechtstheorie wie auch im Lichte des mongolischen Umweltrechts jedoch noch nicht einschätzen.

In einer Gruppe zusammenfassen kann man eine Reihe von Grundrechten zur Ausgestaltung des Arbeits- und Soziallebens. Hierher gehören das Recht auf Bildung, freie Berufsund Arbeitsplatzwahl, "günstige" Arbeitsbedingungen23, Lohn und Erholung sowie das Verbot von Zwangsarbeit 24 , ferner die materielle Absicherung im Alters- und Krankheitsfall wie auch bei Geburt und Kinderpflege. Bemerkenswert ist schließlich das Recht auf

22 Eine gewisse Ungleichbehandlung im alltäglichen Leben kommt indessen zum Ausdruck, wenn Ausländern in Museen höhere Preise abverlangt werden als Einheimischen. Ob dieses, etwa auch im kommunistischen China anzutreffende Vorgehen angesichts der insbesondere von westlichen Touristen erwarteten Wirtschaf tskraft gerechtfertigt ist, mag hier dahingestellt bleiben.

23 Englische Übersetzung: "favourable conditions of work".

24 Auf letzteres beriefen sich auch die Studenten der Mongolischen Staatsuniversität, als sie alten Traditionen entsprechend auch im September 1992 wieder zum staatlich verordneten Emteeinsatz auf das Land mußten. Unter anderem auch durch diesen Einsatz geriet die Kurzzeitdozentur in den Genuß einer gewissen Spontaneität. 
Gesundheitsschutz und auf - im Rahmen konkretisierender Gesetze unentgeltliche - medizinische Betreuung.

In eine weitere Gruppe fallen Rechte, die die Mitwirkung der Bürger an der demokratischen politischen Willensbildung regeln, insbesondere ein umfassendes aktives und passives Wahlrecht und die Parteienfreiheit, ferner das Recht der freien Meinungsäußerung und Information sowie die Versammlungs- und Demonstrationsfreiheit.

Dem Schutz der persönlichen (Bewegungs-) Freiheit und der Privatsphäre dienen vor allem das Verbot willkürlicher Durchsuchungen und Verhaftungen sowie Regeln, die im Falle einer Festnahme einzuhalten sind. Daneben stehen die Unverletzlichkeit der Wohnung sowie das Recht auf Freizügigkeit, Ausreise- und Niederlassungsfreiheit.

Die Bedeutung der garantierten Glaubens- und Gewissensfreiheit mag man sich vergegenwärtigen, indem man an das oft grausame Vorgehen gegen buddhistische bzw. lamaistische Geistliche und Gläubige vor allem in den 30er Jahren unter Marschall Tschoibalsan denkt.

Die bisherige Aufzählung mag einen ersten Eindruck von den unlängst in Kraft gesetzten Grundrechten vermitteln. Tragweite, systematischer Zusammenhang und zugrundeliegendes Grundrechtsverständnis müssen an dieser Stelle indessen noch ebenso unerörtert bleiben wie die Hinlänglichkeit sinnvoller Grundrechtsschranken bzw. in ihren möglichen Konsequenzen zu weitreichende Limitierungen.

\section{Ausblick}

$\mathrm{Ob}$ die eingetretene Entwicklung in Zukunft einen positiven Verlauf finden wird, ist einem sicheren Urteil zwar noch nicht abschließend zugänglich. Allerdings sind eine Reihe von erfolgversprechenden Ansätzen zu erkennen. Dies gilt für den politischen wie für den rechtswissenschaftlichen Bereich.

Im Juni 1992 fanden Parlamentswahlen auf der Basis der neuen Rechtsordnung statt. Daß die alte kommunistische25 MRVP von 76 Sitzen 71 erringen konnte, beruht im wesentlichen wohl auf zwei Gründen. Zum einen waren die neuen Parteien untereinander zerstritten, konnten sich auf ein gemeinsames Vorgehen nicht einigen und wurden als Exponenten des Reformkurses mitunter für die seit Ende der 80er / Anfang der 90er Jahre anhaltende Wirtschaftsmisere verantwortlich gemacht. Schließlich kann das geltende Wahlrecht einer 
kritischen Prüfung kaum standhalten, wenn die angegebene Sitzverteilung bei einem Stimmenverhältnis von ca. $56 \%$ für die MRVP zu $43 \%$ für die anderen Parteien zustande kommt.

Schon dies zeigt, daß eine neue Verfassung allein kein Garant für den Wandel ist Auch fehlt es zur Zeit noch an den institutionellen und rechtsdogmatischen Voraussetzungen ihrer perfekten Umsetzung. Der Aufbau einer am Bild des unabhängigen Richters orientierten Rechtsprechung steckt noch in den Anfängen. Zwar gehen etwa vom Obersten Gericht der Mongolei Impulse in diese Richtung aus. Es bleibt jedoch mit Vorsicht abzuwarten, ob und in welcher personellen Besetzung die bisherigen Änderungen fortgeführt werden können.

Bürgerbeteiligung bei Verwaltungsverfahren und Rechtsschutz gegenüber staatlichen Maßnahmen scheinen dem mongolischen Rechtsbewußtsein momentan noch fremd zu sein. 26 Eine deutschen Verhältnissen entsprechende unabhängige Verwaltungsgerichtsbarkeit ist bislang ebensowenig vorhanden wie eine funktionstüchtige Anwaltschaft nach westlichem Muster.

Die rechtswissenschaftliche Entwicklung wird bisher von drei Institutionen getragen, deren zukünftiges Verhältnis zueinander zur Zeit noch nicht endgültig abzusehen ist. Neben der traditionell überwiegend für die Lehre zuständigen juristischen Fakultät der Mongolischen Staatsuniversität steht die früher so gut wie ausschließlich in der Forschung tätige Akademie der Wissenschaften. In Abkehr von der einstigen strikten Trennung dieser beiden Sphären existiert nun anstelle der Fakultät ein Juristisches Institut, das Universität und Akademie gleichermaßen unterstellt und zugeordnet ist. Außer den genannten Einrichtungen gibt es noch die Akademie für Staats- und Gesellschaftswissenschaften 27 beim Großen Chural. Der ehemaligen Parteihochschule der MRVP könnte die Rolle einer Ausbildungsstätte für qualifizierte Verwaltungsfachleute oder eines wissenschaftlichen Dienstes des Parlaments zufallen. Neben diesen älteren staatlichen Institutionen formiert sich mittlerweile allerdings auch ein auf privater Initiative beruhendes Hochschulwesen.

Indessen steht man hier auf allen Gebieten erst am Anfang der Entwicklung. Doch die Reformbereitschaft der mongolischen Gesprächspartner wirkte generell überzeugend. Vor allem in den jüngeren Generationen der politisch Interessierten und Verantwortlichen ist ein großes fachlich und persönlich beeindruckendes Potential vorhanden.

26 So jedenfalls ist der Eindruck des Verfassers aufgrund der Fragen und Diskussionsbeiträge der Studenten in den Vorlesungen. Ein subjektives öffentliches Recht oder eine drittschützende Norm schien in ihrem Denken nicht zu existieren.

27 Vgl. zur Rolle der Akademie sowie zur allgemeinen wissenschaftlichen Entwicklung auch den Artikel in der FAZ vom 31. Oktober 1992. 
Die Mongolei, etwa viermal so groß wie die Bundesrepublik, durchläuft mit ihren zwei Millionen Einwohnern zur Zeit nicht nur eine Phase der Abkehr vom realen Sozialismus, sondern auch einer Wirtschaftsmisere mit hohen Teuerungsraten, Versorgungsmängeln sowie zunehmender Armut und Kriminalität, einhergehend mit verbreitetem Alkoholismus besonders unter Jugendlichen, denen es an Perspektiven im eigenen Land oft fehlen wird. Die Gefahr, daß dringend benötigte qualifizierte Kräfte abwandern, liegt nahe. Immerhin: Im Gegensatz zu vielen anderen Umwälzungen von Osteuropa bis Zentralasien verlief die Entwicklung in der Mongolei bislang friedlich. Daß berechtigte Hoffnungen zu realen Erfolgen führen, wird nicht zuletzt von der Unterstützung abhängen, die Europa und Nordamerika der jungen Demokratie gewähren. 
is also discussed. The report ends with a number of conclusions of mainly legal character and some recommendations the delegation has addressed to the Austrian government, which, however, may also be of general relevance.

\section{The Economic Constitution of Taiwan - The Situation at the Turning Point}

\section{By Wolfgang Lasars}

Taiwan, that is the Republic of China, understands herself as the anti-communist representative of a free China, on the other hand liberals in Taiwan demand a fundamental marketoriented reform of the Economic Constitution. To understand this contradiction the article examines the Economic Constitution exemplified by the development and the extent of the freedom of enterprises in Taiwan.

On one hand the system of private property and the freedom of enterprises are guaranteed by the Constitution. On the other hand emergency laws which go back to World War II and the civil war against the Chinese Communists have restricted the freedom of enterprises for several decades until 1992. The freedom of enterprises is even more curbed by a large sector of public enterprises and a special sector of corporations owned directly by the ruling party KMT. Because of the dominance of the privileged corporations of the state and the party KMT in the domestic market, many small and medium sized companies have to turn to the export sector. Another related aspect of the Economic Constitution of Taiwan is a large area of illegal but tolerated economic activity. From the beginning onwards, the liberal and democratic opposition in Taiwan has stringly criticized this Economic constitution imposed by the KMT.

Now Taiwan has reached a tuming point: Most probably, the emergency laws will be scrapped, concerning the public corporations the government has initialled first steps in the direction of privatization. However, on the other hand, the corporate sector of the ruling party KMT is still growing. It remains to be seen to which results the present reform process of the Economic Constitution finally will lead.

Mongolia on the Way to Pluralistic Democracy and Market Economy - The New Mongolian Constitution of 1992

\section{By Jürgen Merz}

The communist breakdown in the USSR lead to significant political and constitutional changes in Mongolia as well and initiated a period of transition from totalitarianism and 
centralism to a pluralistic system. Yet, on the other hand, Mongolians place great worth upon their national history and precommunist traditions. At the same time, Mongolia is looking for support from Western democracies. Strong relations to the former German Democratic Republic during the communist era have led to a particularly important role for the Federal Republic of Germany today.

A rather short period of history from the early 13th to the 14th century still forms the source of the national consciousness of present day Mongolia. The dependence on China after this period ended for the first but not the last time in 1911 after the fall of the Manchu dynasty when Mongolian princes and Buddhist priests claimed their sovereignty. Mongolia still remained between Chinese and Russian influences and only gained lasting - formal independence in 1921.

Three years later, the foundation of the Mongolian People's Republic (MPR) started 70 years of communism. The MPR has had three different socialist constitutions dating from 1924, 1940 and 1960. But it was first in 1989 when national oppositions demanded fundamental changes of the political system. Different political parties were then founded and participated in the 1990 elections.

Finally, in 1992 Mongolia got a new constitution based upon the separation of powers doctrine and fundamental human rights. It contains political and economic freedoms as well as provisions for social security. The old nomadic society still dominates the countryside, however, and requires its own property rules. Due to the decades as a Soviet satellite, national independence has been strongly emphasized in the new constitution.

Mongolia faces a deep economic crisis. The survival of the young democracy depends last but not least on strong support from the West. 